\title{
Colour-Doppler features of hepatic involvement in Rendu-Osler-Weber disease
}

\author{
M. Carmen Garre-Sánchez, Francisco Javier Álvarez-Higueras, María Muñoz-Tornero, Akiko Ono-Ono, \\ Manuel Miras-López, José Antonio Pons-Miñano and Luis Fernando Carballo-Álvarez
}

Department of Digestive Diseases. Hospital Universitario Virgen de la Arrixaca. Murcia, Spain

\section{CASE REPORT}

A 39-year-old woman with a past history of Rendu-Osler-Weber disease of 16 years was referred to our unit to evaluate hepatic involvement. Previous pulmonary arteriovenous malformations were treated with embolization. It is known that recurrence of pulmonary arteriovenous malformations (VMs) requires a new treatment modality. On physical examination no skin lesions were found. Laboratory parameters were normal. Ultrasound imaging findings were: Dilation of hepatic artery (diameter $12 \mathrm{~mm}$ ), tortuous intrahepatic structures, tubular parallel structures to the portal branches and a shunt between hepatic artery and left portal vein. Spleen size was normal (Figs. 1-3).

\section{DISCUSSION}

Hereditary haemorrhagic telangiectasia (HHT), or Rendu-Osler-Weber disease, is an autosomal dominant vascular disorder of the fibrovascular tissue that is characterized by widespread mucocutaneous telangiectasia and VMs that commonly occur in the pulmonary, hepatic and cerebral circulations. Hepatic involvement is found in up to 41-84\% patients (1) and it is represented by a spectrum of vascular abnormalities, which evolve from tiny telangiectasias to substantial vascular malformations, which may progress to larger arteriovenous shunt. Symptoms of hepatic VMs generally do not occur before the age of 50. Strong and significant predominance of VMs in female patients has been observed. The majority of patients are asymptomatic, however, symptoms related to these lesions can include: Heart failure, portal hypertension and biliary disease, reflecting different patterns of vascular involvement. Diagnosis of liver involvement in HHT can be made by Doppler-colour ultrasound in asymptomatic patients and seems to provide a favourable argument for screening for liver VMs (2). Imaging findings include (1-3):

- An increased diameter of the hepatic artery $(>7 \mathrm{~mm})$.

- Tortuous intrahepatic tubular structures.

- Tubular structures parallel to the portal branches representing dilated arterial branches.

- Vascular shunts.

Doppler-US screening should be performed in asymptomatic patients and angio-TC or angio-RM should be performed to detect VMs in other organs or if high quality ultrasound is not available (2).

No treatment is recommended for HHT patients with asymptomatic liver involvement. In HHT patients with symptomatic liver disease a multidisciplinary approach for management and treatment is required. Invasive treatments aims to reduce the shunt by surgical ligation or transarterial embolization, however both treatment approaches should

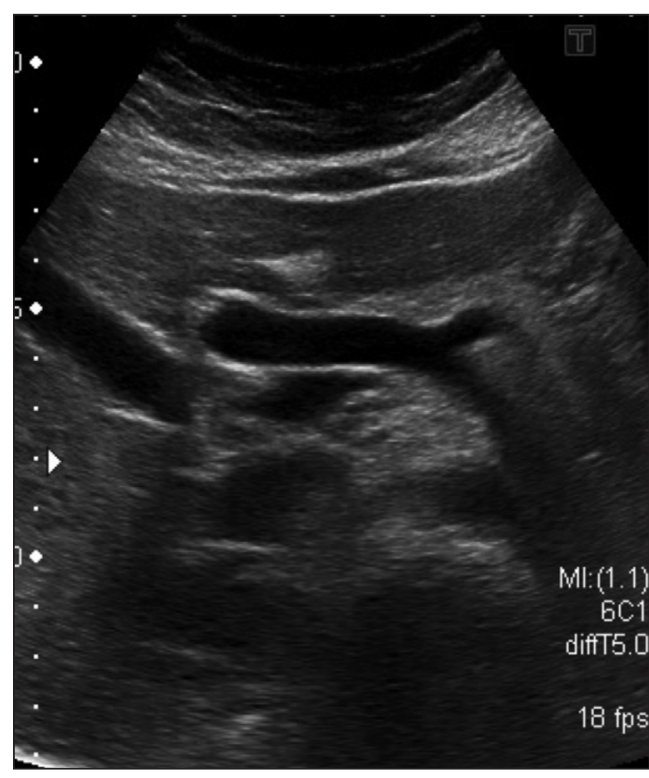

Fig. 1. Celiac trunk and dilated hepatic artery $(12 \mathrm{~mm})$ 
be considered mainly in patients who are not candidates for liver transplantation and failed medical therapy due to risk of complications (4).

Systemic treatment with the vascular endothelial growth factor Bo(VEGF)-inhibitor, bevacizumab, have reduced nasal bleeding and improved cardiac insufficiency (5).

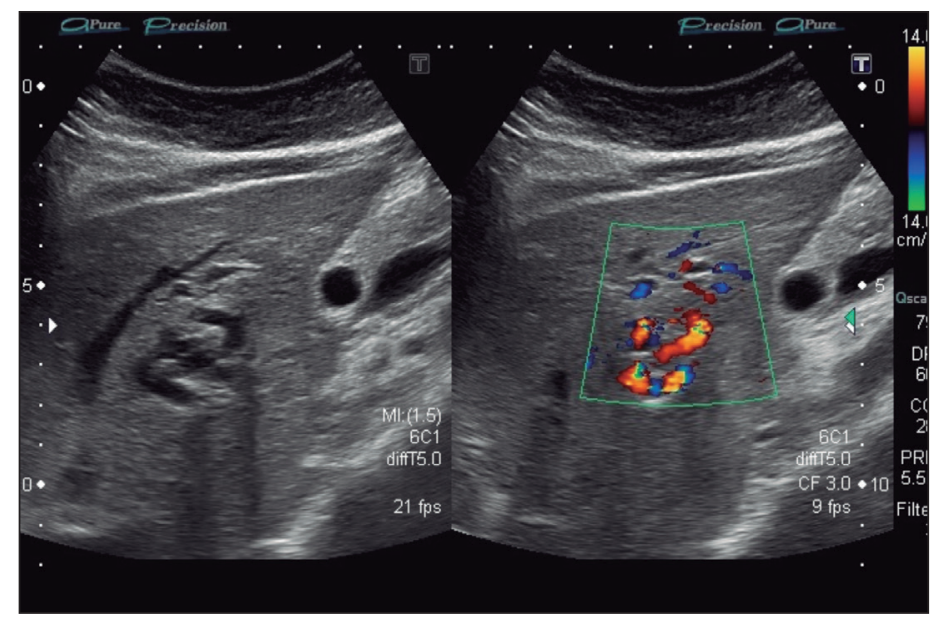

Fig. 2. Intrahepatic arteriovenous malformations.

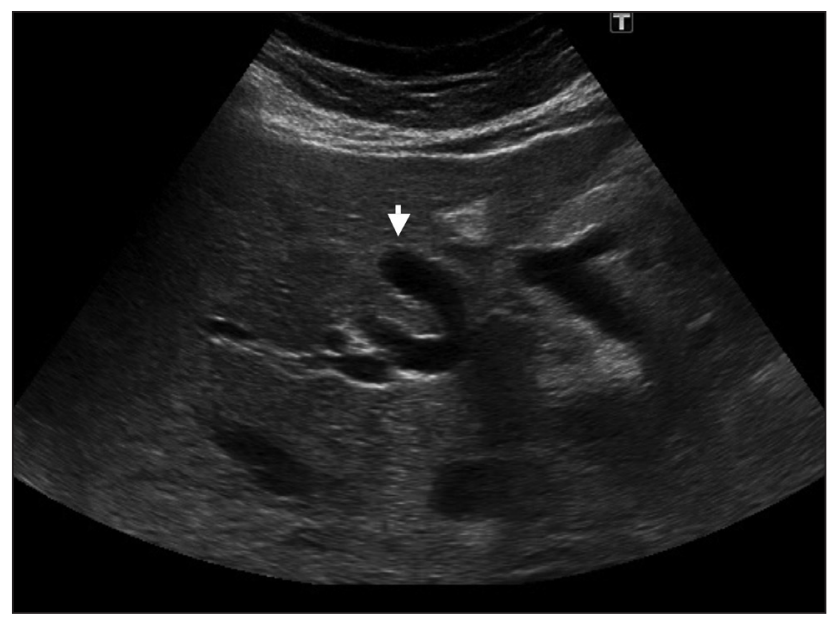

Fig. 3. Arteriovenous fistula between hepatic artery and left portal vein.

\section{REFERENCES}

1. Sabbà C, Pompili M. Review article: The hepatic manifestations of hereditary haemorrhagic telangiectasia. Aliment Pharmacol Ther 2008;28:523-33.

2. Buscarini E, Leandro G, Conte D, Danesino C, Daina E, Manfredi G, et al. Natural history and outcome of hepatic vascular malformations in a large cohort of patients with hereditary hemorrhagic teleangiectasia. Dig Dis Sci 2011;56:2166-78.

3. Deftereos S, Kranou L, Michailidou E, Prassopoulos P. Hepatic arteriovenous malformation in Rendu-Osler-Weber syndrome. J Gastrointestinal Liver Disease 2012;21:109-10.

4. Núñez Viejo MA, Fernández Montes A, Hernández Hernández JL, Pons Romero F, Fábrega García E, Zarrabeitia R. Enfermedad de Rendu-Osler con afectación hepática: primer trasplante en España. Med Clin 2010;135:552-5.

5. Dupuis-Girod S, Chesnais AL, Ginon I, Dumortier J, Saurin JC, Finet G, et al. Long-term outcome of patients with hereditary hemorrhagic telangiectasia and severe hepatic involvement after orthohepatic liver transplantation: A single-center study. Liver Transplantation 2010;16:340-7. 